\title{
Radiative corrections in a minimal extension of the standard model.
}

\author{
J. J. van der Bija国 \\ ${ }^{a}$ Fakultät für Mathematik und Physik, Physikalisches Institut, Universität Freiburg, \\ H. Herderstr. 3, 79104 Freiburg i.B., Germany
}

Radiative corrections are studied within an extension of the standard model, containing extra singlet scalars. The calculations determine the effect of a large width of the Higgs boson on radiative corrections. They throw some light on the treatment of unstable particles inside loop-graphs.

\section{INTRODUCTION}

The standard model of elementary particle physics based on the gauge group $S U(3) \times$ $S U(2) \times U(1)$ is essentially confirmed by the precision tests at LEP [ 1]. However the Higgs particle has not been found and a naive analysis of the data gives a bound of $m_{H}>112 \mathrm{GeV}$. However the data are not without trouble. There is a large difference between the leptonic data, including the measured $\mathrm{W}$-mass and the results from the b-physics data at LEP. The leptonic data by themselves would imply a lower mass for the Higgs boson than is acceptable from the direct search. While it is probably premature to conclude that the standard model is definitely ruled out, the discrepancy points out the importance of studying alternatives to the minimal Higgs sector of the standard model.

Because the effect is small and the general agreement with the standard model is there, a natural option is to study the effects of changes in the Higgs sector alone. The simplest such extension is to include extra Higgs singlet scalar particles in the model. Singlets do not couple to ordinary matter directly in a renormalizable way, but only to the Higgs sector. Therefore indirect effects of such particles appear only at the twoloop level in precision tests of the standard model. Since effects at the two-loop level are generically suppressed, significant contributions of the sin-

\footnotetext{
*This work was supported by the European Union under contract HPRN-CT-2000-00149 and by the DFGForschergruppe: Quantenfeldtheorie, Computeralgebra und Monte-Carlo-Simulation.
}

glets can only arise if they have at least moderately strong interactions. When this is the case one can in principle not limit oneself to two-loop graphs only, but needs to resum an infinite number of graphs, thereby being forced to confront the non-convergent nature of perturbation theory. This problem is not unique to Higgs physics, but arises always when one has instable particles inside loops, since the propagator of an instable particle implies a Dyson-resummation of the oneparticle irreducible contribution to the two-point function. In gauge theories this leads to complications regarding gauge invariance and questions regarding high precision predictions for instance for the process $e^{+} e^{-} \rightarrow W^{+} W^{-}$.

In order to address such questions it is advantageous to have a simple model, that avoids some of the complications. Also the presence of a non-perturbative expansion parameter is desirable. These arguments lead one to consider the standard model coupled to an $\mathrm{O}(\mathrm{N})$-symmetric sigma-model[ 2]. By a suitable rescaling of coupling constants one can use the parameter $(1 / \mathrm{N})$ as a non-perturbative expansion parameter. It is this model that will be used in the following. Besides the fact that it is essentially the simplest extension of the standard model and therefore should be studied, the model has some interesting features. If the extra scalars are light enough, the Higgs boson will decay into them, giving a large invisible width to the Higgs boson. This will eliminate practically any signal at the LHC. However TESLA [3] will have no problem discovering such a Higgs boson. The decay products of the Higgs boson are stable and weakly interacting with or- 
dinary matter. They form a suitable candidate

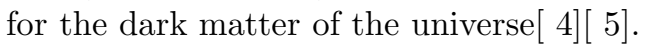

\section{MNMSM or STEALTH MODEL}

The model of a Higgs sector, containing the standard model Higgs boson plus an $\mathrm{O}(\mathrm{N})$ symmetric sigma model, is essentially the simplest extension of the standard model and one can call it the $\mathrm{M}$ (inimal) $\mathrm{N}$ (on)-M(inimal) $\mathrm{S}$ (tandard) $\mathrm{M}$ (odel), filling in the gap between SM, MSSM and NMSSM. Because of the hidden nature of the Higgs boson one can also call it the stealth model.

The Lagrangian density is the following:

$$
\begin{aligned}
L_{\text {Scalar }}= & L_{\text {Higgs }}+L_{\text {Phion }}+L_{\text {Interaction }} \\
\text { where } & \\
L_{\text {Higgs }}= & -\partial_{\mu} \phi^{+} \partial^{\mu} \phi-\lambda\left(\phi^{+} \phi-\frac{v^{2}}{2}\right)^{2} \\
L_{\text {Phion }}= & -\frac{1}{2} \partial_{\mu} \vec{\varphi} \partial^{\mu} \vec{\varphi}-\frac{1}{2} m_{P}^{2} \vec{\varphi}^{2}-\frac{\kappa}{8 N}\left(\vec{\varphi}^{2}\right)^{2} \\
L_{\text {Inter. }}= & -\frac{\omega}{2 \sqrt{N}} \vec{\varphi}^{2} \phi^{+} \phi
\end{aligned}
$$

Here we use a metric with signature $(-+++)$. $\phi=\left(\sigma+v+i \pi_{1}, \pi_{2}+i \pi_{3}\right) / \sqrt{2}$ is the complex Higgs doublet of the SM with the vacuum expectation value $\langle 0|\phi| 0\rangle=(v / \sqrt{2}, 0), v=246 \mathrm{GeV}$. Here, $\sigma$ is the physical Higgs boson and $\pi_{i=1,2,3}$ are the three Goldstone bosons. $\vec{\varphi}=\left(\varphi_{1}, \ldots, \varphi_{N}\right)$ is a real vector with $\langle 0|\vec{\varphi}| 0\rangle=\overrightarrow{0}$. We consider the case, where the $O(N)$ symmetry stays unbroken, because we want to concentrate on the effects of a finite width of the Higgs particle. Breaking the $O(N)$ symmetry would lead to more than one Higgs particle, through mixing. Since the goal of this exposition is to clarify the role of a finite width of the Higgs boson inside loop graphs, the model will be analyzed in the limit, where the phion mass is zero. Also the phion selfinteractions will be ignored. In this limit fully analytic results can be presented. A detailed analysis of the general case, which necessarily becomes numerical, is given in [6]. The physics of the model in the above limit is quite simple. One has a Higgs boson that has a larger than usual width, but there are no other direct physics effects present. Within the $1 / N$ expansion only bubble-graphs containing phions contribute to the Higgs propagator, which enters the electroweak radiative corrections.

\section{THE $\rho$ PARAMETER}

\subsection{Definition}

Higgs mass dependent corrections to low energy electroweak parameters, appear in a number of places. Because the Yukawa couplings are very small, the Higgs dependent effects are limited to corrections to the vector-boson propagators, which can be taken to be the $\rho$ parameter, the mass shift of the $\mathrm{Z}$ boson and the mass shift of the $\mathrm{W}$ boson, often parametrized by $\mathrm{S}, \mathrm{T}, \mathrm{U}$ or $\epsilon_{1}, \epsilon_{2}, \epsilon_{3}$ [ [7]. The behaviour for the different quantities is very similar. If the Higgs mass is much larger than the Z-boson mass, the effects grow like $\log \left(m_{H}^{2} / M_{W}^{2}\right)$. Already for the present limit $m_{H}>112 \mathrm{GeV}$, the large Higgs mass limit is a reasonable approximation. As the behaviour for the different quantities is similar, it is sufficient to focus on one of them. We will here choose the socalled $\rho$ parameter. It is the ratio of neutral to charged current strengths. The definition is:

$\rho=G_{F}^{0} / G_{F}^{+}$

At the tree level $\rho=1$. This is peculiar for a doublet Higgs field, the reason being the $O(4)$ symmetry of the Higgs potential, which is larger than the $S U(2) \times U(1)$ symmetry of the standard model as as whole. This larger symmetry is broken by the hypercharge coupling, leading to effects vanishing with $\operatorname{tg}\left(\theta_{W}\right)$. The correction is given by:

$\delta \rho=\rho-1=\left(\delta M_{W}^{2}-\cos ^{2}\left(\theta_{W}\right) \delta M_{Z}^{2}\right) / M_{W}^{2}$

where $\delta M_{W}^{2}$ and $\delta M_{Z}^{2}$ are the corrections to the vector boson masses at $k^{2}=0$.

\section{2. $\delta \rho$ in the standard model}

Within the standard model the Higgs mass dependent contribution can be most easily described in the unitary gauge, where only onegraph contributes, namely the virtual splitting and recombining of a vectorboson into a Higgs boson and a vectorboson. This holds for all the low energy precision variables, which explains why 
the contributions are so similar. This contribution, coming from the Higgs alone is still divergent, the divergence being canceled by graphs containing only virtual vectorbosons and photons. The exact expression becomes quite complicated. For the Higgs mass dependent contribution it is therefore advantageous to consider the difference with the hypothetical case of $m_{H}=0$. In the limit, that the Higgs mass becomes large one then finds the following simple result:

$$
\begin{array}{r}
\delta \rho\left(S M, m_{H}\right)-\delta \rho\left(S M, m_{H}=0\right)=-\frac{3 g^{2}}{64 \pi^{2}} \\
\left(t g^{2}\left(\theta_{W}\right) \log \left(m_{H}^{2} / m_{Z}^{2}\right)+\log \left(1+t g^{2}\left(\theta_{W}\right)\right)\right.
\end{array}
$$

The last term comes from the fact that one compares diagrams with different masses inside the loops; it is actually $\log \left(m_{Z}^{2} / m_{W}^{2}\right)$. If one is interested in just the leading logarithm, without the constant, one can make an expansion directly inside the graphs and ignore the masses of the vectorbosons. One finds:

$$
\begin{array}{r}
\delta \rho=\frac{g^{2}}{\left(2 \pi^{4}\right) i} t^{2}\left(\theta_{W}\right)(1-1 / n) \\
\int d^{n} k\left(k^{2}\right)^{-1}\left(k^{2}+m_{H}^{2}\right)^{-1}
\end{array}
$$

This gives the leading Higgs mass behaviour. The integral is still divergent, but this divergence is canceled by the explicit vectorboson graphs. The extra factor $\left(k^{2}\right)^{-1}$ simplifies the integral, which is useful in particular when more loops are present.

\section{FINITE WIDTH EFFECTS}

\subsection{Perturbation theory}

We are now ready to calculate higher order effects in the model coming from the extra interactions of the Higgs boson to the phions. Within the $1 / N$ expansion the only contributions that arise are graphs with phion bubbles inserted in the Higgs propagator. The calculations can therefore be performed in the same style as the one-loop calculations. Contributions to the $\rho$ parameter at the n-phion bubble level are therefore determined by integrals of the form:

$\int d^{4} k \frac{1}{k^{2}} \frac{1}{\left(k^{2}+m_{H}^{2}\right)^{n+1}} \log ^{n}\left(k^{2} / m_{H}^{2}\right)$
The logarithms in this integral come from the phion-bubbles, that are easily calculated, since they are just the massless one-loop propagator graphs. The one-loop renormalization of the Higgs mass is taken into account, through the scale choice of $\mu=m_{H}$ in the bubble-graphs. Going to polar coordinates and using the simplification due to the factor $\left(k^{2}\right)^{-1}$ it is now relatively easy to calculate the diagrams. The result is:

$\delta \rho=\frac{3 g^{2} \operatorname{tg}^{2}\left(\theta_{W}\right)}{64 \pi^{2}} \sum_{n=1}^{\infty} \int_{0}^{\infty}(-\Delta)^{n} \frac{\log ^{n}(s)}{(s+1)^{n+1}} d s(7)$

where:

$\Delta=\frac{\omega^{2} v^{2}}{32 \pi^{2} m_{H}^{2}}$

Explicit evaluation of the integrals gives:

$\delta \rho=\frac{3 g^{2} t g^{2}\left(\theta_{W}\right)}{64 \pi^{2}} \sum_{n=2}^{\infty} \frac{(-2 \pi \Delta)^{n}}{n}\left|B_{n}\right|\left(1-2^{1-n}\right)(9)$

where $B_{n}$ are the Bernouilli numbers. Since the odd Bernouilli numbers are 0, only the graphs with an even number of phion-bubbles contribute. This at least partly explains the observation [\&], that at the two-loop level within the standard model the correction due to the Higgs particle is small. It was noticed, that the correction is small, not only because of the loop-factors, but also because of extra cancellations between different terms in the final result. The above result explains that there is no enhancement proportional to the number of Goldstone bosons, three in the standard model.

\subsection{Borel summation}

The series that we found for the $\rho$ parameter is obviously divergent. The question is therefore in how far one can make sense of the series. A traditional way to look at such a series is to make a Borel transformation. The Borel sum is defined as follows. Given the above series

$\sum_{n=2}^{\infty} a_{n} \Delta^{n}$

we form the new "Borel" series

$F(z)=\sum_{n=2}^{\infty} a_{n} z^{n-1} /(n-1)$ ! 
We find :

$F(z) \sim \frac{\pi}{\sin (\pi z)}-\frac{1}{z}$

If the Borel transformed function $F(z)$ would have no singularities on the positive real axis, one could construct a regular function $G(\Delta)$ having the correct perturbation expansion. One takes the exponentially damped integral:

$G(\Delta)=\int_{0}^{\infty} \exp (-y / \Delta) F(y) d y$

However in our case the Borel transform has an infinity of poles for positive values of the coupling constant $\Delta$. This means that there is no unambiguous way to resum the perturbative series, so that non-perturbative effects much be present. In order to get a meaningful result one has to deform the contour in the Borel plane. The correct choice of the contour is in general arbitrary and some physical insight is necessary. Here the situation is very similar to the calculation of the one loop effective Lagrangian in an external electric field in QED. The contour is deformed so as to give an imaginary part to the effective Lagrangian, which has the physical interpretation, that the electric field is instable under decay into electron-positron pairs. In the approximation we used in the calculation, the vacuum also ought to be instable, since in the bubblesum only the term $H \vec{\varphi}^{2}$ contributes, which corresponds to a potential that is unbounded from below.

In complete analogy we therefore define the resummed series for $\delta \rho$ by:

$\delta \rho=\int_{0}^{-i \infty} \exp (-y / \Delta) F_{\rho}(y) d y$

This way one find an imaginary part :

$\operatorname{Im}(\rho)=\frac{3 g^{2} t g^{2}\left(\theta_{W}\right)}{64 \pi^{2}} \frac{-i \pi}{\exp (1 / \Delta)+1}$

The non-perturbative form of the contribution is manifest. Actually one can evaluate the complete contribution to $\delta \rho$ :

$$
\begin{aligned}
& \delta \rho=\frac{-3 g^{2} t g^{2}\left(\theta_{W}\right)}{64 \pi^{2}} \\
& (\psi(1 / 2-i /(2 \pi \Delta))+\log (2 \pi \Delta)+i \pi / 2)
\end{aligned}
$$

Here the $\psi$-function is the logarithmic derivative of the $\Gamma$-function. For large width $\Delta \rightarrow \infty$ one has the following asymptotic expansion:

$$
\begin{aligned}
& \delta \rho=\frac{-3 g^{2} t g^{2}\left(\theta_{W}\right)}{64 \pi^{2}} \\
& \left(\gamma_{\text {Euler }}+2 \log (2)+\log (2 \pi \Delta)+i \pi / 2\right)
\end{aligned}
$$

This shows, that if the Higgs is light, but has a large width, the width plays the same role as a large Higgs mass, as far as low energy radiative corrections are concerned.

\subsection{Direct summation}

While the approach described above is mathematically acceptable, it is not quite satisfactory from the physical point of view. The presence of an imaginary part to $\delta \rho$ is clearly an artefact of the approximation, namely the effective use of a cubic potential. Within the full theory this term cannot be there, as the potential is positive definite. Also the separate summation of the higher-loop graphs, does not emphasize the connection between loop-graphs and corresponding tree graphs. A more naive calculation would consist of summing the bubbles in the Higgs propagator first and then substituting the resummed propagator into the loop-graph.

If one does this, one can consider the correction to the $\rho$-parameter as an averaged correction due to a density of Higgs-fields. The density is the Kallén-Lehmann mass density $\sigma(s)$ of the Higgs propagator. In formula-form:

$\delta \rho=\int d s \sigma(s) \delta \rho\left(m_{H}^{2}=s\right)$

In this from the origin of the problem gets localized in the fact that the resummed Higgs propagator contains a tachyon pole $m_{T}^{2}=-s_{0} m_{H}^{2}$ at:

$s_{0}+1+\Delta \log \left(s_{0}\right)=0$

The residue at the pole is given by $-s_{0} /\left(\Delta+s_{0}\right)$. The presence of a tachyon is unacceptable and one has to find a prescription to deal with it. Simply subtracting the pole part is reasonable, as it leads to a Kallén-Lehmann density $\sigma(s)$ that is physical in the sense that $\sigma$ has support only for positive s. However it leads to an undefined value of the 
$\rho$-parameter. The reason for this is that the integral $\int d s \sigma(s)$ is not unity anymore. One has to correct for this effect. The simplest way is to add a non-perturbative factor $\left(\Delta+s_{0}\right) / \Delta$ to the Higgs-propagator. This last correction factor is of course somewhat arbitrary, as one needs only an overall normalization of the integral of $\sigma$. This kind of uncertainty appears to be unavoidable, since one is not able to solve the theory non-perturbatively. As the effect is however suppressed by a factor $\exp (-1 / \Delta)$ the effect is very small in the case of a normal small coupling. The maximum correction factor we get here is in the case $\Delta=1$ and is about $28 \%$. A graph of the correction factor as a function of $\Delta$ is given in figure 1 .

\section{CONCLUSION}

In conclusion, we have discussed the problem of an unstable Higgs particle inside loops, both by a direct summation and by substituting a resummed propagator in the loop. In both cases an uncertainty in the result is present, due to the fact that we cannot solve the theory nonperturbatively. The uncertainty was quantified to be maximally around $28 \%$. Therefore qualitatively correct results can still be derived even for a wide Higgs. In this case the radiative effects cannot be distinguished from the effects of a heavy Higgs particle. The width starts playing the role of the Higss mass, which is easy to understand on the basis of the Kallén-Lehmann representation. We therefore conclude, that this model and similar ones with a wide mass density cannot explain the dicrepancies in the precision data, which were the original motivation of this study. The model is not better, but also not worse than the standard model.

While the method appears to work well in the present simple situation, a number of open questions remain. Foremost is the question, whether this method can be applied to gauge-theories. Furthermore one should consider the possibility of going to higher orders in the $1 / N$ expansion. Some work along these lines, within the heavyHiggs standard model, has been performed in [ 9][ 10]. Finally connections with fully non- perturbative methods such as the lattice would be of interest.

\section{REFERENCES}

[1] S. Riemann, in this proceedings (2002).

[2] T. Binoth and J.J. van der Bij, Z. Phys. C75 (1997) 17.

[3] TESLA, Technical Design Report, Part III (2001) 46.

[4] M.C. Bento, O. Bertolami and R. Rosenfeld, Phys. Lett. B518 (2001) 276.

[5] C.P. Burgess, M. Pospelov and T. ter Veldhuis, Nucl. Phys. B619 (2001) 709.

[6] R. Akhoury, J.J. van der Bij and H. Wang, Eur. Phys. J. C20 (2001) 497.

[7] Review of Particle Physics, Eur. Phys. J. C15 (2000) 104.

[8] J.J. van der Bij and M.J.G. Veltman, Nucl. Phys. B231 (1984) 205.

[9] T. Binoth, A. Ghinculov and J.J. van der Bij, Phys. Lett. B417 (1998) 343.

[10] A. Ghinculov, T. Binoth and J.J. van der Bij, Phys. Rev. D57 (1998) 1487. 


$$
\begin{aligned}
& \mathrm{m}_{\varphi}=0 \\
& \tilde{\kappa}=0
\end{aligned}
$$

$\begin{array}{ll}1.0 & 2.0 \quad \Delta\end{array}$

$3.0 \quad 4.0$

Figure 1. Non-perturbative propagator correction factor. 